\title{
Evaluación de la microdureza superficial de una resina compuesta según fuente de luz, su opacidad y tiempo de exposición
}

Webb-Linares LJ, Reynoso-Zeballos GE, Manuel Lagravere-Vich M, Delgado-Cotrina LA. Evaluación de la microdureza superficial de una resina compuesta según fuente de luz, su opacidad y tiempo de exposición. Rev Estomatol Herediana. 2009; 19(2):96-102.

\section{RESUMEN}

El objetivo de este estudio fue comparar la microdureza superficial de una resina compuesta según el tipo de lámpara o fuente de luz, opacidad y tiempo de exposición. Se utilizaron la lámpara halógena Astralis ${ }^{\circledR} 10$ (Ivoclar Vivadent) y la lámpara LED BluePhase ${ }^{\circledR}$ (Ivoclar Vivadent). Se utilizó la resina microhíbrida 4Season ${ }^{\circledR}$ (Ivoclar Vivadent) en los colores Enamel Trans Super Clear (ETSC), Enamel A2 (A2E), Dentin A2 (A2E). El tiempo de exposición empleado fue de $20 \mathrm{~s}$, $40 \mathrm{~s}$ y $60 \mathrm{~s}$. Se confeccionaron 5 muestras de $5 \times 5 \times 2 \mathrm{~mm}$ por cada grupo de estudio. Las muestras se almacenaron durante 24 horas en un recipiente seco y oscuro después de la polimerización. Las muestras se evaluaron utilizando la prueba de microdureza Vickers con un microdurómetro HMV-2 (Shimadzu, Kyoto, Japón), en la superficie superior e inferior. Los datos fueron analizados a través de la prueba ANOVA/Tukey. Para detectar diferencias entre las superficies se empleó el análisis t de Student para muestras pareadas. Los mayores valores de microdureza superficial fueron para la resina Enamel Trans Super Clear. La lámpara halógena proporcionó mayores valores de microdureza superficial. Para las lámparas LED o hubo diferencia significativa a los 20s, 40s y 60s. Para la lámpara halógena a los 20s se obtuvo los menores valores de microdureza; entre 40s y 60 s no hubo diferencia significativa.

Palabras clave: PRUEBAS DE DUREZA / RESINAS COMPUESTAS.

Microhardness evaluation of composite resin by light source, opacity and exposure time

ABSTRACT

The aim of this study was to compare the surface microhardness of a composite resin according to the light source, opacity and time of exposure. The halogen lamp Astralis ${ }^{\circledR} 10$ (Ivoclar Vivadent), BluePhase ${ }^{\circledR}$ LED lamp (Ivoclar Vivadent), and a microhybrid composite resin 4Season (Ivoclar Vivadent) in colors Enamel Trans Super Clear (ETSC), Enamel A2 (A2E), Dentin A2 (A2E) were used. The exposure times used were 20s, 40s and 60s. For each group, five samples of $5 \times 5 \times 2 \mathrm{~mm}$ were made. After the polymerization, the samples were stored for 24 hours in a dry and dark container. The samples were evaluated using Vickers microhardness test through the HMV-2 microhardmeter (Shimadzu, Kyoto, Japan) on the upper and lower surface. The data were analyzed by ANOVA test (Tukey). The student's t paired samples was used to detect differences between the surfaces. The resin Enamel Super Clear Trans has the highest values for surface microhardness. Halogen lamp supplies the highest values of surface microhardness. For the LED lamp there was not significant difference at 20s, 40s and 60s.The halogen lamp at 20s obtain lowest values for surface microhardness; between $40 \mathrm{~s}$ and $60 \mathrm{~s}$ there were not significant difference.

Key words: HARDNESS TESTS / COMPOSITE RESINS.

\section{Introducción}

Son muchos los factores que influyen en el éxito o fracaso de una restauración con resina compuesta. Uno de ellos es la polimerización, donde se busca convertir la mayor cantidad de monómeros en polímeros ya que de esto dependerán las propiedades de la restauración y las complicaciones asociadas a esta (13). Una inadecuada polimerización de las resinas compuestas puede generar propiedades físicas inferiores, fallas de retención, mayor solubilidad y respuestas pulpares adversas por la presencia de una gran cantidad de monómeros no polimerizados (4).

El tipo de fuente o lámpara utilizada, el espectro de emisión de luz, la intensidad de luz emitida y la técnica de polimerización son factores asociados con la eficiencia de polimerización de las resinas activadas por luz $(4,5)$.

La lámpara halógena ( $\mathrm{LH}$ ) consta de un foco halógeno que emite luz dentro del espectro de luz visible y un filtro que reduce el espectro entre 400 y 500nm. A pesar de su popularidad estas lámparas presentan muchas limitaciones tales como la reducción gradual de la salida de energía debido a la
Louise Jacqueline Webb

Linares $^{1}$

Giovanna Elizabeth Reynoso

Zeballos $^{2}$

Manuel Lagravere Vich $^{3}$

Leyla Antoinette Delgado Cotrina ${ }^{1}$

Docente del Departamento Académico de Clínica del Adulto. Facultad de Estomatología. Universidad del Adulto. Facultad de Estom
Peruana Cayetano Heredia.

'Cirujano-Dentista, Magíster en Estomatología. ${ }^{3}$ Investigador Asociado. Facultad de Estomatología. Universidad de Alberta, Canadá.

\section{Correspondencia}

Louise Jacqueline Webb Linares Av. Honorio Delgado 430-Lima 31, Perú Teléfono: (511) 381-1950

e-mail: ljacquelinewebbl@ hotmail.com

Recibido : 12 de mayo del 2009

Aceptado : 15 de julio del 2009 degradación del filtro y foco, profundidad de polimerización limitada, largo tiempo de exposición y vida útil reducida, calculada aproximadamente entre 40 y 100 horas (6-8). Para superar estas limitaciones Mills et al. (9) propusieron el uso de Diodos Emisores de Luz (LED). Los diodos son dispositivos electrónicos que sirven para controlar el flujo de corriente eléctrica y son construidos con materiales semiconductores (silicio, germanio, arseniato de galio, etc.). Un LED tras un estímulo de energía eléctrica la transforma en energía electromagnética en una 
determinada longitud de onda.

Entre las ventajas de las lámparas LED podemos citar: mayor tiempo de vida útil (10000 horas), no necesitan filtros y tamaño reducido. Las primeras lámparas fabricadas con esta tecnología emitían baja intensidad de luz por lo cual no podían utilizarse en tiempos clínicamente aceptables (7). Por otro lado, la luz se emitía en un estrecho rango de longitud de onda, entre 450 y 500nm, longitud de onda que es óptimo para la alcanforquinona (CQ), sin embargo, es inadecuado para otro tipo de fotoiniciadores con espectros de absorción de luz que oscilan entre los 400 a 450nm o menos. Nuevas generaciones de LEDs se han lanzado al mercado con mejoras sustanciales que permiten su uso en la práctica clínica $(10,11)$.

Diversos estudios que han empleado LEDs para polimerizar resinas se reportan en la literatura (10-17). Algunos demuestran similar eficacia cuando se compara con las lámparas halógenas $(11,15-17)$ y otros afirman que esta tecnología falta desarrollarse $(13,14)$ pero casi todos concuerdan que es una tecnología potencial para este fin (18).

Por otro lado, el color de las resinas compuestas puede influir en la polimerización. Para Martins et al. (19) el color no tiene mucha influencia sobre la polimerización y para Pereira (20) el color no tiene mucha influencia cuando la intensidad de luz es superior a $300 \mathrm{~mW} / \mathrm{cm} 2$.

Sin embargo, Delgado (10) encontró que las resinas extraclaras presentan menores valores de microdureza que el color A2, argumentando que este fenómeno está relacionado con la mayor opacidad de las resinas extraclaras.
La opacidad de la resina puede influir en la polimerización de las resinas compuestas y afectar directamente la profundidad de polimerización. Ésta va a depender del color del material y del grosor del mismo (21).

Otro factor a considerar es el tiempo de exposición, Nomoto et al. (22) encontraron que cuando la densidad de energía (intensidad de luz x tiempo de exposición) es constante la profundidad de polimerización y grado de conversión son similares demostrando una influencia de la intensidad y el tiempo. David et al. (11) evaluaron la influencia del tiempo de exposición encontrando un aumento significativo de la microdureza superficial de las resinas compuestas evaluadas cuando se aumentó el tiempo de exposición de 20 a 40 segundos.

Conocer como cada factor influye en las propiedades de una restauración es importante para poder aplicar los materiales en la situación más indicada. Así, este trabajo busca evaluar la influencia de la fuente de luz, opacidad y el tiempo de exposición en la microdureza superficial de una resina compuesta.

\section{Material y métodos}

Las fuentes utilizadas se muestran en la Tabla 1. La resina evaluada fue 4 Season ${ }^{\circledR}$ (Ivoclar Vivadent, Liechtenstein) colores Enamel Transparent Super Clear (ETSC), A2 esmalte (A2E), A2 dentina (A2D) cuyos grados de translucidez son 56\%; 13,7\%; 7,9\% respectivamente (23). El tiempo de exposición utilizado fue de 20, 40 y 60 segundos. La Tabla 2 muestra los grupos experimentales. Cada grupo tuvo cinco muestras.

Para la confección de las muestras se emplearon matrices metálicas de $5 \mathrm{~mm}$ de diámetro y

Tabla 1. Características de lámparas usadas.

\begin{tabular}{lllcc}
\hline Lámpara & $\begin{array}{l}\text { fuente } \\
\text { de luz }\end{array}$ & fabricante & $\begin{array}{c}\text { intensidad de } \\
\text { luz }\left(\mathrm{mw} / \mathrm{cm}^{2}\right)\end{array}$ & $\begin{array}{c}\text { longitud de } \\
\text { onda }(\mathrm{nm})\end{array}$ \\
\hline Astralis 10 & Halógena & Ivoclar-Vivadent & 650 & $400-510$ \\
Bluephase & LED & Ivoclar-Vivadent & 650 & $430-490$ \\
\hline
\end{tabular}

Tabla 2. Grupos experimentales.

\begin{tabular}{lllc}
\hline Grupo & color de resina & tipo de fuente & tiempo (s) \\
\hline G1 & SC & LH & 20 \\
G2 & SC & LH & 40 \\
G3 & SC & LH & 60 \\
G4 & SC & LED & 20 \\
G5 & SC & LED & 40 \\
G6 & SC & LED & 60 \\
G7 & A2E & LH & 20 \\
G8 & A2E & LH & 40 \\
G9 & A2E & LH & 60 \\
G10 & A2E & LED & 20 \\
G11 & A2E & LED & 40 \\
G12 & A2E & LED & 60 \\
G13 & A2D & LH & 20 \\
G14 & A2D & LH & 40 \\
G15 & A2D & LH & 60 \\
G16 & A2D & LED & 20 \\
G17 & A2D & LED & 40 \\
G18 & A2D & LED & 60 \\
\hline
\end{tabular}


2mm de profundidad $(4,10)$. Se utilizó platinas de vidrio para obtener superficies lisas, y para que no existan variaciones en la microdureza de la superficie inferior, siempre se trabajó sobre una superficie de cartulina negra $(14,24)$.

El tiempo de exposición fue monitoreado con un cronómetro digital. Inmediatamente después de polimerizar las muestras fueron envueltas en papel aluminio y almacenadas secas en un recipiente oscuro durante 24 horas.

Transcurrido este tiempo se realizó la prueba de microdureza Vickers $(10,11,17,25)$ utilizando un microdurómetro HMV-2 (Shimadzu, Kyoto, Japón) usando una carga de 50 gf durante 30s $(10,11,17,25)$. Se realizaron cuatro impresiones en la superficie superior (superficie más cercana a la fuente de luz) y 4 en la superficie inferior (superficie más alejada a la fuente de luz) $(10,17,25)$.

Una vez recolectados los datos, fueron ingresados y analizados en el programa estadístico SPSS versión 13.0.

Se realizó estadística descriptiva (media y desviación estándar). Para determinar las diferencias de medias entre los grupos se empleó el análisis de varianza (ANOVA/Tukey), con un nivel de significancia de 95\%.

\section{Resultados}

La media y la desviación estándar de los valores de microdureza se muestran en la Tabla 3. Según el análisis de varianza, la interacción entre el color y la fuente de polimerización fue estadísticamente significativa con respecto a la microdureza obtenida $(\mathrm{p}=0,0109)$ para la superficie superior.

Para la superficie inferior las interacciones entre la opacidad $\mathrm{x}$ tiempo, $(\mathrm{p}=0,0222)$; opacidad $\mathrm{x}$ fuente $(\mathrm{p}=0,0013)$ y tiempo $\mathrm{x}$ fuente $(p=0,0209)$ fueron estadísticamente significativas. Para comparar los valores de microdureza entre las superficies se utilizó la prueba t de Student para muestras pareadas, mostrando que los valores de microdureza en la superficie superior fueron mayores que en la superficie inferior $(p<0,05)$. Para los grupos 11 y 12 esta diferencia no fue estadísticamente significativa.

La fuente de luz influyó en los valores de microdureza en función de la opacidad y del tiempo. La resina ETSC obtuvo los mayores valores de microdureza cuando fue polimerizada con LH. En relación al tiempo de exposición se puede observar que cuando el tiempo fue de 20s los valores obtenidos con LH y LED fueron similares en cambio, cuando se incremento el tiempo a 40 s ó 60 s se obtuvo mayor microdureza con LH. Independientemente del color utilizado, cuando se utilizo lámpara LED no se encontró diferencias entre 20s, 40s y 60s (Fig. 1).

\section{Discusión}

En este estudio se analizó la influencia del tipo de fuente, la opacidad y el tiempo de exposición sobre la microdureza superficial de una resina compuesta.

Según el análisis de los datos se encontró que el tipo de fuente influyó en los resultados siendo que $\mathrm{LH}$ proporcionó mayores valores de microdureza, excepto para la resina A2E y A2D a 20s. Para A2D esta diferencia fue estadísticamente significativa. Estos resultados concuerdan con los hallazgos de Hoffman et al. (5), Delgado (10) y Uhl et al. (14). Estas diferencias encontradas pueden estar relacionadas con la intensidad de luz y la composición de las resina compuestas.

Kilian en 1979 (26) observó un aumento de microdureza conforme aumenta la intensidad de la luz. Por otro lado, Nomoto et al. (22) encontraron que cuando la densidad

Tabla 3.Media y desviación estándar de los valores de microdureza Vickers de la superficie superior y de la superficie inferior.

\begin{tabular}{|c|c|c|c|c|c|}
\hline Grupo & color & $\begin{array}{l}\text { fuente } \\
\text { de luz }\end{array}$ & tiempo & $\begin{array}{l}\text { superficie } \\
\text { superior }\end{array}$ & $\begin{array}{l}\text { superficie } \\
\text { Inferior }\end{array}$ \\
\hline$\overline{\mathrm{G} 1}$ & SC & $\mathrm{LH}$ & 20 & $56,70(5,19) g$ & 45,43 (3,28)g,h \\
\hline G2 & SC & LH & 40 & 53,13 (2,37)e,f,g & $48,19(2,32) \mathrm{h}$ \\
\hline G3 & SC & LH & 60 & $58,25(5,46) g$ & $50,86(5,81) h$ \\
\hline G4 & SC & LED & 20 & 52,43 (3,67)e,f,g & 30,43 (2,07)a,b,c \\
\hline G5 & SC & LED & 40 & $55,23(2,6) \mathrm{g}$ & 36,46 (2,65)b,c,d,e \\
\hline G6 & SC & LED & 60 & 53,43 (1,37)f,g & $37,34(2,1) c, d, e, f$ \\
\hline G7 & $\mathrm{A} 2 \mathrm{E}$ & LH & 20 & 51,04 (3,77)d,e,f,g & 30,07 (2,52)a,b \\
\hline G8 & A2E & LH & 40 & $52,72(2,82) e, f, g$ & 40,81 (1,37)e,f,g \\
\hline G9 & A2E & $\mathrm{LH}$ & 60 & $57,72(3,51) \mathrm{g}$ & 43,78 (5,47)f,g,h \\
\hline G10 & $\mathrm{A} 2 \mathrm{E}$ & LED & 20 & $45,53(2,67)$ c,d,e & $37,16(5,43) b, c, d, e, f$ \\
\hline G11 & A2E & LED & 40 & 35,06 (3,74)a,b & 33,22 (0,91)b,c,d \\
\hline G12 & A2E & LED & 60 & $36,04(2,01) a, b$ & 34,82 (0,91)b,c,d,e \\
\hline G13 & A2D & $\mathrm{LH}$ & 20 & $30,75(3,30) a$ & $23,56(3,22) a$ \\
\hline G14 & A2D & LH & 40 & $39,79(2,96) b, c$ & 36,46 (2,89)b,c,d,e \\
\hline G15 & A2D & LH & 60 & $38,54(2,72) b, c$ & 35,41 (3,17)b,c,d,e \\
\hline G16 & A2D & LED & 20 & $39,23(1,81) b, c$ & $32,59(1,45) \mathrm{b}, \mathrm{c}, \mathrm{d}$ \\
\hline G17 & A2D & LED & 40 & $44,13(4,22) c, d$ & 34,99 (2,35)b,c,d,e \\
\hline G18 & A2D & LED & 60 & 45,73 (3,94)c,d,e,f & 37,74 (2,09)d,e,f \\
\hline
\end{tabular}

Nota: Letras minúsculas diferentes en cada columna indican diferencia estadísticamente significativa entre los grupos $(\mathrm{p}<0,05)$ 


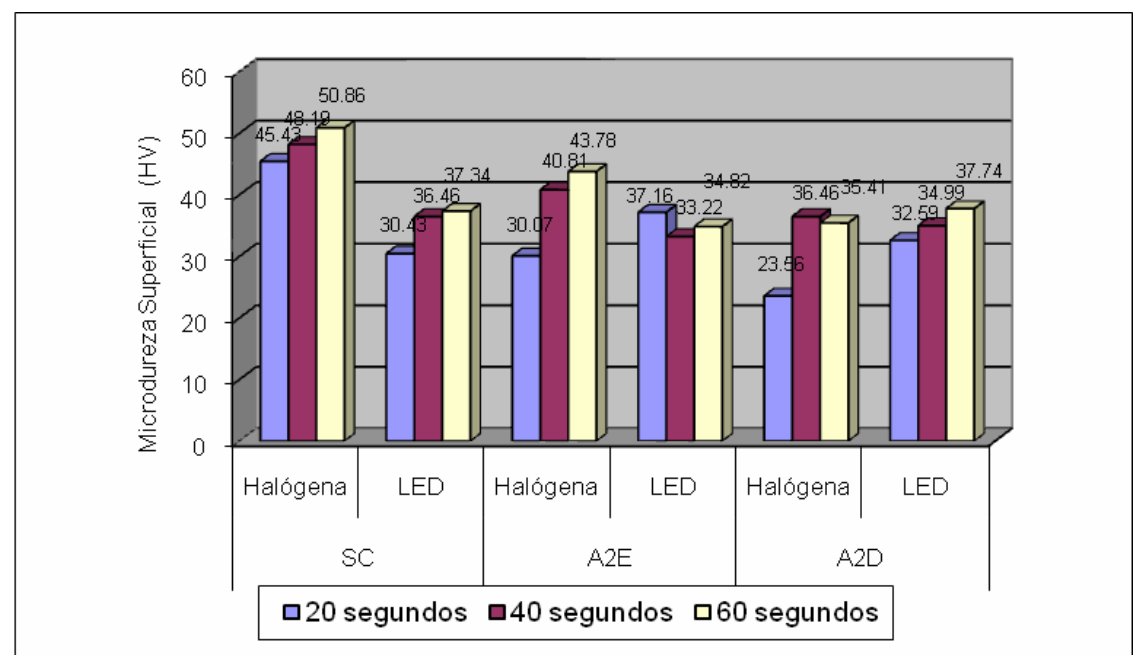

Fig. 1. Valores de microdureza Vickers de la superficie superior y de la superficie inferior según fuente de luz, opacidad y tiempo de exposición.

de energía (intensidad de luz $\mathrm{x}$ tiempo) es constante los resultados de profundidad de polimerización y grado de conversión son semejantes. Para el presente estudio la intensidad de luz de la lámpara Astralis 10 y la Bluephase fue de $650 \mathrm{~mW} / \mathrm{cm} 2$.

Para Hofmann et al. (5) LH y LED con alta intensidad producen similar microdureza en resinas que contienen solo alcanforquinona mientras que para las resinas que tienen un fotoiniciador adicional encontraron bajos valores de microdureza después de ser irradiados con LED.

Gritsch et al. (27) encontraron mayores valores de microdureza con LH que con LED para resinas microhíbridas y nanohíbridas. Ellos notaron que la resina Tetric EvoCeram (Ivoclar) contiene 2 fotoiniciadores (Alcanforquinona y Lucerina TPO) a diferencia de la resina Ceram X (Dentsply) que contiene solo Alcanforquinona. Basados en sus resultados concluyeron que las diferencias entre LH y LED son menos importantes en la resina que contiene un fotoiniciador que cuando contiene dos.

Sin embargo, Correr et al. (28) y el pasaje de luz (29).

Martins et al. (19) utilizaron resinas con diferentes matices y con la misma saturación (A3, B3, C3, D3 e I). Determinaron que el color no influye en los resultados de microdureza como otros factores. Pereira (20) afirmó que el color no tuvo influencia cuando la intensidad de luz es mayor a los $300 \mathrm{~mW} / \mathrm{cm} 2$. Sin embargo, encontró que el color C4 presentó mayores valores de microdureza que el color A1 cuando el tiempo de exposición fue de 60 segundos. Similares resultados obtuvieron Dunn y Bush (4) cuando compararon el color B1 y C4, donde el color C4 tuvo mayores valores de microdureza cuando fue polimerizado con LH. Delgado (10) comparó diferentes resinas color A2 y su respectivo color extraclaro encontrando que los colores extraclaros presentaron menores valores de microdureza.

Estos hallazgos podrían explicarse debido al tipo de pigmento incorporado en cada color de resina lo cual repercute en la opacidad de la misma. En los estudios de Dunn y Bush (4) y Pereira (20) se compararon pigmentos rojo marrón y amarillo respectivamente con resinas con pigmento gris. Según Hirata et al. (30) las resinas con mayor cantidad de pigmentos blancos son más opacas que las resinas con pigmentos grises los cuales tienen una mayor tendencia a la translucidez.

Los menores valores de microdureza de las resinas A2E y A2D pueden estar relacionados al grado de opacidad y a la composición de las mismas. En relación a la composición, se sabe que las resinas extraclaras o translucidas tienen menor cantidad de CQ e incorporan otros fotoiniciadores debido al color 
amarillo de este fotoinicador. Los fotoiniciadores como fenilpropanodiona (PPD) o Lucerina tienen el potencial de reducir los problemas de color asociados a las resinas al ser más claros o modifican su color al completar la polimerización $(11,18,31)$. Estos fotoiniciadores en combinación con la CQ actúan sinérgicamente para producir una polimerización más eficiente.

Esto tiene importancia cuando se elije el tipo de fuente a utilizar ya que el espectro de absorción de estos fotoiniciadores es diferente al de la CQ Para la Lucerina el pico de absorción está alrededor de 370nm (14) y para el PPD 405nm (11) hecho que explicaría los mayores valores de microdureza de la resina ETSC cuando se polimerizó con LH. Para el factor tiempo de exposición se puede apreciar que cuando se polimerizó con LH no hubo diferencia en los valores obtenidos con 20, 40 y 60s a excepción de la resina A2E y A2D que obtuvieron menor microdureza cuando se polimerizó a 20s. Para el caso de las LED no existió diferencia en la polimerización entre 20, 40, 60s.

Turbino et al. (32) evaluaron la relación entre la microdureza Vickers con el tiempo de exposición (20s y 40s). Encontraron que el tiempo de exposición de 40s proporcionó los mayores valores de microdureza independientemente de la resina compuesta utilizada.

David et al. (11) encontraron que las LED son tan eficientes como las LH para polimerizar las resinas Charisma SL y Tetric Ceram XL en 40 s o más. Concluyen que el tiempo de exposición de 40s es necesario para conseguir una microdureza uniforme cuando se emplea LED.

Della Bona et al. (33) estudiaron la influencia del color y el tiempo de irradiación sobre la dureza de las resinas compuestas, refieren que sólo los grupos expuestos a un tiempo de polimerización de 40s fueron capaces de producir adecuados valores de dureza promedio para ambas superficies superior e inferior para los mismos especímenes. Sin embargo, encontraron diferencias significativas entre la superficie superior e inferior. Recomiendan que para lograr una adecuada dureza a lo largo de un incremento de $3 \mathrm{~mm}$ de espesor de resina compuesta el clínico debe fotoactivar durante 40 segundos.

En el presente estudio, para todos los grupos la microdureza fue mayor en la superficie superior que en la inferior excepto en el grupo G11 y G12 (Esmalte A2 polimerizado durante 40s y 60s con lámpara LED). Con estos hallazgos podemos verificar la reducción de valores de microdureza en las zonas más profundas. Estos hallazgos concuerdan con los resultados de Knezevic et al. (1), David y et al. 11), Gomes et al. (17), Rastelli (25), Hansen et al. (34), Arikawa et al. (35). Esto está directamente relacionado a la cantidad de luz incidente o intensidad de luz. La superficie superior está en contacto con la punta activa lo que significa una mayor cantidad de luz o fotones efectivos para reaccionar con los fotoiniciadores. La superficie inferior se encuentra alejada de la punta activa por lo que la luz tendrá que atravesar el material y por fenómenos de absorción y dispersión de la luz existen menos fotones disponibles para reaccionar con los iniciadores, por lo tanto, existe una menor conversión de monómero a polímeros que se manifiesta en una disminución en la microdureza (36).
Como vemos, son muchos los factores que pueden influir en la polimerización de las resinas compuestas. Muchos esfuerzos se hacen para disminuir las complicaciones sin embargo, es necesario conocer como estos pueden influir en nuestra práctica clínica e interactuar con los materiales y equipos que disponemos. Futuras investigaciones deben ser realizadas para conocer mejor como actúan los diferentes factores.

\section{Agradecimientos}

Al laboratorio de Ingeniería de Materiales de la Universidad Estadual de Ponta Grossa, Paraná, Brasil, donde se realizaron las medidas de microdureza.

A la casa dental E. B. Pareja Lecaros S. A., Lima, Perú, por facilitar las resinas y las lámparas utilizadas en el estudio.

\section{Referencias bibliográficas}

1. Knezevic A, Tarle Z, Meniga A, Sutalo J, Pichler G, Ristic M. Degree of conversion and temperature rise during polymerization of composite resin samples with blue diodes. J Oral Rehabil. 2001; 28(6):586-91.

2. Fan PL, Schumacher RM, Azzolin K, Geary R, Eichmiller FC. Curing-light intensity and depth of cure of resin-based composites tested according to international standards. J Am Dent Assoc. 2002; 133(4):42934.

3. Cunha LG, Alonso RC, Pfeifer CS, Correr-Sobrinho L, Ferracane JL, Sinhoreti MA. Contraction stress and physical properties development of a resin-based composite irradiated using modulated curing methods at two C-factor levels. Dent 
Mater. 2008; 24(3):392-8.

4. Dunn WJ, Bush AC. A comparison of polymerization by light-emitting diode and halogenbased light-curing units. J Am Dent Assoc. 2002; 133(3):33541.

5. Hofmann N, Hugo B, Klaiber B. Effect of irradiation type (LED or QTH) on photo-activated composite shrinkage strain kinetics, temperature rise, and hardness. Eur J Oral Sci. 2002;110(6):471-9.

6. Santos MJMC, Souza Jr MHS, Mondelli RFL. Novos conceitos relacionados à fotopolimerização das resinas compostas. J Bras Dent Estet. 2002; 1(1): 14-21.

7. Mills RW, Uhl A, Jandt KD. Optical power outputs, spectra and dental composite depths of cure, obtained with blue light emitting diode (LED) and halogen light curing units (LCUs). Br Dent J. 2002; 193(8):459-63.

8. 3M-ESPE ELIPARTM 2500 Halogen Curing Light: Technical Product Profile. 2003.

9. Mills RW, Jandt KD, Ashworth SH. Dental composite depth of cure with halogen and blue light emitting diode technology. $\mathrm{Br}$ Dent J. 1999; 186(8):388-91.

10.Delgado C. Avaliação da microdureza superficial de resinas compostas extraclaras fotopolimerizadas por luz halógena e LEDs. Descrição das características dos aparelhos fotopolimerizadores. [Tesis de maestría]. 2004. Universidade Estadual de Ponta Grossa Brasil. 130p.

11. David JR, Gomes OM, Gomes JC, Loguercio AD, Reis A. Effect of exposure time on curing efficiency of polymerizing units equipped with light-emitting diodes. J Oral Sci. 2007; 49(1):19-24.

12. Swift EJ Jr. Visible light-curing. J Esthet Restor Dent. 2001; 13(1):16-7.

13.Kurachi C, Tuboy AM, Magalhães DV, Bagnato VS. Hardness evaluation of a dental composite polymerized with experimental LED-based devices. Dent Mater. 2001; 17(4):309-15.

14.Uhl A, Mills RW, Jandt KD. Photoinitiator dependent composite depth of cure and Knoop hardness with halogen and LED light curing units. Biomaterials. 2003; 24(10):178795.

15.Tsai PC, Meyers IA, Walsh LJ. Depth of cure and surface microhardness of composite resin cured with blue LED curing lights. Dent Mater. 2004; 20(4):364-9.

16. Wiggins KM, Hartung M, Althoff O, Wastian C, Mitra SB. Curing performance of a newgeneration light-emitting diode dental curing unit. J Am Dent Assoc. 2004; 135(10):1471-9.

17. Gomes GM, Calixto AL, Santos FA, Gomes CM, D'Alpino PH, Gomes JC. Hardness of a bleaching-shade resin composite polymerized with different light curing sources. Pesqui Odontol Bras. 2006;20(4):337-41.

18. Aravamudhan K, Floyd CJ, Rakowski D, Flaim G, Dickens SH, Eichmiller FC, Fan PL. Light-emitting diode curing light irradiance and polymerization of resin-based composite. J Am Dent Assoc. 2006; 137(2):21323.

19. Martins F, Delben AC, Santos LR, Soares, HL, Martins E Ede O. Microdureza de resina em função da core luz halógena.
Pesqui Odontol Bras. 2002; 16(3):246-250.

20.Pereira SK. Resina composta fotopolimerizável. Avaliação da dureza superficial em função de: cor, tempo de exposição, intensidade de luz e profundidade do material. [Tesis de Doctorado]. Araraquara (Brasil): Universidade Estadual Paulista Araraquara; 1999.

21. Miyagawa Y, Powers JM, O'Brien WJ. Optical properties of direct restorative materials. J Dent Res. 1981; 60(5):890-4.

22. Nomoto R, Uchida K, Hirasawa T. Effect of light intensity on polymerization of light-cured composite resins. Dent Mater J. 1994; 13(2):198-205.

23.Ivoclar Vivadent. 4Seasons ${ }^{\circledR}$. Documentación científica. Mayo - 2003.

24. Uhl A, Sigusch BW, Jandt KD. Second generation LEDs for the polymerization of oral biomaterials. Dent Mater. 2004; 20(1):80-7.

25. Rastelli ANS Avaliação da profundidade de polimerização de uma resina composta, pela técnica trans-dental, utilizando-se três diferentes fontes de luz: halógena, laser de argônio e LEDs. [Tesis de Maestría]. São Paulo (Brasil): Universidade Estadual Paulista; 2002.

26. Kilian RJ. Visible light cured composites: dependence of cure on light intensity [abstract]. J Dent Res. 1979; 58:243. Abstract 603.

27.Gritsch K, Souvannasot S, Schembri C, Farge P, Grosgogeat $B$. Influence of light energy and power density on the microhardness of two nanohybrid composites. Eur J Oral Sci. 2008; 116(1):77-82.

28.Correr AB, Sinhoreti MA, 
Sobrinho LC, Tango RN, Schneider LF, Consani S. Effect of the increase of energy density on Knoop hardness of dental composites light-cured by conventional QTH, LED and xenon plasma arc. Braz Dent J. 2005; 16(3):218-24.

29. Kawaguchi M, Fukushima T, Miyazaki K. The relationship between cure depth and transmission coefficient of visible-light-activated resin composites. J Dent Res. 1994; 73(2):516-21.

30. Hirata R, Ampessan EL, Liu J. Reconstrução de dentes anteriores com resinas compostas - Uma seqüência de escolha e aplicação de resinas. J Brasil Clin Estet Odonto. 2001; 5(25):15-25.

31.Park YJ, Chae KH, Rawls HR. Development of a new photoinitiation system for dental light-cure composite resins. Dent Mater. 1999; 15(2):120-7.

32.Turbino ML, Vinha D, Centola AL, Campos GM. Photopolymerized resins: surface hardness variation in relation to time of polymerization and setting. Braz Dent J. 1993; 3(2):87-94.

33. Della Bona A, Rosa V, Cecchetti D. Influence of shade and irradiation time on the hardness of composite resins. Braz Dent
J. 2007; 18(3):231-4.

34. Hansen EK, Asmussen E. Correlation between depth of cure and temperature rise of a light-activated resin. Scand J Dent Res. 1993; 101(3):176-9.

35. Arikawa H, Kanie T, Fujii K, Fukui K, Homma T. Mechanical properties of light-cured composite resins cured through filters that simulate enamel. Dent Mater J. 2002; 21(2):147-55.

36.Rueggeberg FA, Craig RG. Correlation of parameters used to estimate monomer conversion in a light-cured composite. J Dent Res. 1988; 67(6):932-7. 